\title{
Germanica
}

\section{" Pris en flagrant délit » : ces lieux hantés par l'interdit dans La bascule du souffle de Herta Müller}

"Auffrischer Tat ertappt": Das Verbotene in Herta Müllers Atemschaukel "Auf frischer Tat ertappt": Forbidden Spaces in Herta Müller's The Hunger Angel

Michel Mallet

\section{OpenEdition}

\section{Journals}

Édition électronique

URL : http://journals.openedition.org/germanica/4737

DOI : $10.4000 /$ germanica.4737

ISSN : 2107-0784

Éditeur

Université de Lille

Édition imprimée

Date de publication : 31 juillet 2018

Pagination : 127-140

ISBN : 9782913857414

ISSN : 0984-2632

Référence électronique

Michel Mallet, « «Pris en flagrant délit » : ces lieux hantés par l'interdit dans La bascule du souffle de Herta Müller », Germanica [En ligne], 62 | 2018, mis en ligne le 31 juillet 2020, consulté le 15 janvier 2021. URL : http://journals.openedition.org/germanica/4737 ; DOI : https://doi.org/10.4000/ germanica. 4737 


\title{
« Pris en flagrant délit »: ces lieux hantés par l'interdit dans La bascule du souffle de Herta Müller
}

\author{
Michel MALLET \\ Université de Moncton
}

Que ce soit dans ses récits autobiographiques ou dans son œuvre de fiction, l'interdit se retrouve souvent au cœur des thématiques abordées par Herta Müller. Celui-ci - par nature étroitement lié à la tradition, à la morale et aux lois - est couramment contextualisé et associé au concept identitaire propre aux cultures germanophones que l'on appelle Heimat. Espace à la fois sentimental et physique prônant la normativité, la Heimat que décrit Müller dans ses œuvres se veut aussi un lieu où règnent les divisions, notamment celles liées aux discours opposant le bien et le mal, le permis et le proscrit, la vérité et le mensonge. Toutefois, au risque de se voir menacés, punis et exclus de leur communauté, rares sont les protagonistes de Müller qui se plient au protocole de conduite construit et prescrit par la Heimat. Reflétant l'expérience de l'auteure en tant que victime de dictature et de surveillance ${ }^{1}$, les personnages roma-

1. - À ce sujet, Johannsen explique que « Müller est sans conteste une auteure politique dont l'écriture décrit et met en évidence les mécanismes de surveillance et d'oppression et leurs effets sur les personnes ». « Müller is without question a political author whose writing describes and indicts the mechanisms of surveillance and oppression and their effects on people». Anja Johannsen, « Osmoses : Müller's Things, Bodies, and Spaces », in : Bettina Brandt, Valentina Glajar (dir.), Herta Müller : Politics 
nesques de Müller sont eux aussi prédestinés à la clandestinité, à un mode de vie marginal à la fois préventif et stratégique qui entraîne peur et hantise. Dans leur ouvrage Secret Spaces, Forbidden Places, Fran Lloyd et Catherine O'Brien observent les liens qui unissent les lieux de clandestinité au phénomène de l'interdit :

Le secret peut inclure la suppression de la vérité, la dissimulation d'informations ou l'enfouissement des désirs ou des pensées sombres, que ce soit par des individus, des groupes ou des gouvernements. Habitant les labyrinthes de la ville, enfouis dans le corps, dans les voûtes des archives ou encore dans les profonds recoins de l'esprit, le secret est à la fois l'espace et le lieu à travers lesquels se manifeste l'interdit ${ }^{2}$.

Tel que le décrivent Lloyd et O'Brien dans ce passage, l'interdit est intrinsèquement lié au domaine du dissimulé, au monde du secret. Bien que le secret et l'interdit occupent une place de prédilection dans les romans de Müller, il n'en reste pas moins que la corrélation liant l'espace, la clandestinité et la surveillance est particulièrement visible dans son roman La bascule du souffle 3 (2009), où l'identité refoulée et les vices cachés du protagoniste et narrateur Léo Auberg font de lui une proie des mécanismes de surveillance de la Heimat. Incapable de résister à la tentation de ses désirs, il est à la fois obsédé par l'angoisse de se trahir et séduit par les lieux de l'interdit qu'il ne peut s'empêcher de fréquenter, malgré la menace constante de la surveillance, ce risque d'être pris en flagrant délit.

\section{La surveillance de la Heimat}

Fondé sur la vie du poète roumain Oskar Pastior avec qui Müller a initialement projeté d'écrire le roman, La bascule du souffle raconte l'histoire du jeune Léo qui, soixante ans plus tard, se souvient de ses 17 ans et plus particulièrement de ce soir de janvier 1945, date fatidique à laquelle il est déporté dans un camp de travaux forcés en Ukraine. Il se

and Aesthetics, Lincoln and London, University of Nebraska Press, 2013, p. 208.

2. - « The secret may include the suppression of truth, the concealment of information or the preservation of desires or dark knowledge, whether by individuals, groups, or governments. Inhabiting the city's labyrinths, embedded in the body, buried in the vaults of the archive or the deep recesses of the mind, the secret is both the space and the site through which, and upon which, the forbidden operates. » Par Fran Lloyd et Catherine O'Brien, Secret spaces, forbidden places, New York, Oxford, Berghahn Books, 2000, p. xvi dans l'introduction.

3. - Herta Müller, La bascule du souffle, roman traduit de l'allemand par Claire de Oliveira, Paris, Éditions Gallimard, 2010, cité par la suite sous la forme BS. Les traductions dans cette publication proviendront de : Herta Müller, Atemschaukel, München, Hanser, 2009, cité par la suite sous la forme AS. 
rappelle aussi que sa peur d'être puni pour son homosexualité refoulée était plus grande que celle de se trouver sur la liste des germanophones de Transylvanie devant être déportés en URSS, tel qu'exigé par le nouvel allié soviétique de Bucarest en cette période de fin de guerre. Pour l'adolescent en pleine fleur de l'âge, l'angoisse d'être déporté au camp de travaux forcés, ce lieu habité par la mort, n'est certes pas encore tangible. Mais le désir, voire le besoin de quitter la Heimat, lui, est déjà bel et bien présent :

[Je l'avais] accepté en me disant, du haut de mes dix-sept ans, que ce départ arrivait à point nommé. La liste russe, j’aurais certes pu m'en passer, mais partir me ferait vraiment du bien, à moins que ça tourne au vinaigre. Je voulais quitter ma petite ville, ce dé à coudre où toutes les pierres avaient des yeux ${ }^{4}$. (BS, 10)

Habité par la peur grandissante d'être surveillé par tous et par tout dans cette Heimat qu'il décrit comme étant un lieu "où même les pierres [avaient] des yeux $»^{5}(\mathrm{BS}, 9)$, Léo veut à tout prix fuir cet espace hanté par l'interdit.

Ainsi, le panoptique, ce concept construit autour du principe de contrôle et d'espionnage en milieu carcéral, peut s'avérer intéressant et utile dans l'analyse du phénomène de hantise de la surveillance qui afflige les personnages de Müller, surtout en ce qui a trait au jeune protagoniste de La bascule du souffle. Dans Le Pouvoir psychiatrique, Michel Foucault propose la théorie selon laquelle

[le] vrai effet du Panopticon [est] d'être tel que, même lorsqu'il n'y a personne, l'individu dans sa cellule, non seulement se croie, mais se sache observé. [...] Pas besoin d'armes, de violences physiques, de contraintes matérielles. Mais un regard qui surveille et que chacun, en le sentant peser sur lui, finira par intérioriser au point de s'observer lui-même: chacun, ainsi, exercera cette surveillance sur et contre lui-même ${ }^{6}$.

S'intéressant tout comme Foucault à cette idée du regard à la fois extérieur et intérieur qui surveille tout en mode continu, Gary Shapiro s'inspire lui aussi du concept du panoptique pour décrire ce qu'il nomme, dans son ouvrage intitulé Archeologies of Vision, le « generalized

4. - « [Ich habe] mir gedacht mit meinen siebzehn Jahren, dass dieses Wegfahren zur rechten Zeit kommt. Es müsste nicht die Liste der Russen sein, aber wenn es nicht zu schlimm kommt, ist es für mich sogar gut. Ich wollte weg aus dem Fingerhut der kleinen Stadt, wo alle Steine Augen hatten » (AS, p. 7).

5. - «wo alle Steine Augen hatten » (ibid.).

6. - Michel Foucault, Le Pouvoir psychiatrique - Cours au Collège de France. 1972-1973, Paris, Gallimard, 2003, p. 78. 
occular regime $»^{7}$, une forme de surveillance omniprésente dérivée du « mauvais œil » et que l'on peut parfois retrouver dans certaines sociétés patriarcales. Propre à une communauté conservatrice qui, soucieuse de protéger les codes de conduite de ses membres, guette tout, partout et en tout temps, cette forme de surveillance correspond à la description que fait le narrateur des mécanismes de surveillance dont il est témoin dans sa Heimat natale, cet endroit confiné et confinant qu'il compare d'ailleurs à un « Fingerhut », un dé à coudre (BS, 10). Sous l'impression des regards qui le surveillent, il se sent étouffé par ce que la théorie du panoptique décrit comme étant « cette surveillance [de l'individu] sur et contre lui-même $»^{8}$. Demeurant aux aguets, contraint de camoufler son identité et ses gestes, il devient de plus en plus reclus, de plus en plus « eingesperrt », enfermé (AS, 321).

Par le biais de Léo et de son homosexualité refoulée, ce sentiment de culpabilité que le protagoniste a su garder sous silence, Müller expose encore une fois les contraintes normatives et conservatrices de la Heimat. Le système binaire d'inclusion et d'exclusion sur lequel celleci se fonde a pour effet d'étouffer tout ce qui ne correspond pas à son idéal normatif, puisque la différence, l'altérité, sont perçues comme des tares, des vices qui ternissent l'image sereine et soi-disant innocente de la Heimat. Or, tel que le décrit Peter Blickle dans son ouvrage Heimat: A Critical Theory of the German Idea of Homeland, prétendre l'innocence n'est pas la même chose qu'être innocent ${ }^{9}$. Cette observation de Blickle correspond à la position anti-Heimat de Müller, position qu'elle défend en se fondant sur le caractère à la fois trompeur et dangereux qu'elle associe à ce concept identitaire qu'elle juge par ailleurs nationaliste et idéologique. Ainsi, Müller fait souvent référence, dans ses essais biographiques, à la duplicité de la Heimat, notamment dans « Heimat oder der Betrug der Dinge » (1997) où elle la compare entre autres à un «Versteckspiel ${ }^{10}-$ un jeu de cache-cache où règnent l'hypocrisie et le mensonge. Car bien que sa fonction soit de projeter une image de pureté

7. - Gary Shapiro, Archeologies of Vision: Foucault and Nietzsche on seeing and saying, Chicago, University of Chicago Press, 2003, p. 8.

8. - M. Foucault, Le pouvoir psychiatrique, op. cit., p. 78.

9. - Blickle explique que «[...] l'invocation d'une idée qui est associée à l'innocence n'est pas la même chose que l'innocence. Et cela montre comment l'idéalisation de l'espace territorial associé à la Heimat a souvent causé la création de frontières d'exclusion. » « [...] the invocation of an idea that is associated with innocence is not the same as innocence. And it shows how the idealization of a home ground in Heimat has led again and again to borders of exclusion. » Peter Blickle, Heimat - A Critical Theory of the German Idea of Homeland, Rochester, Camden House, 2002, p. 158.

10. - Herta Müller, Heimat oder Der Betrug der Dinge. Kein Land in Sicht: Heimat - weiblich?, München, Ed. Gisela Ecker, Fink, 1997, p. 214. 
et d'innocence, la Heimat se veut une construction sociale opérant sur des mécanismes d'inclusion et d'exclusion. Par conséquent, la communauté de la Heimat a facilement recours aux reproches, aux accusations et à l'humiliation pour éloigner celles et ceux qui ne se conforment pas à ses normes sociales définies et catégoriques. Conscient d'avoir rompu avec les codes hétéro-normatifs imposés par sa communauté, Léo perçoit son identité refoulée comme étant la tare qui l'aura privé d'un réel sentiment d'appartenance à la Heimat.

\section{La honte des vices cachés}

Victime à la fois de sa propre homophobie et de celle de sa communauté, accablé par des sentiments de confusion et de honte, Léo se rappelle le besoin urgent de camoufler ce que lui-même percevait comme étant un défaut impur et déplorable, un vice interdit qui devait à tout prix demeurer caché, loin des regards scrutateurs de sa communauté. Il se souvient de la façon dont son sentiment de culpabilité l'a poussé à éprouver du dégoût et de la honte envers sa propre orientation sexuelle, lorsqu'il souligne : « [j']avais eu des histoires. Interdites. Ç’avait été bizarre, sale, impudique et beau »11 (BS, 8). Tel qu'exposé par le biais des souvenirs de sa jeunesse, il est conscient que l'engagement dans des actes sexuels connotés comme impurs entacherait les valeurs de sa famille et de sa communauté, lesquelles n'hésiteraient pas à le condamner, à l'humilier et à le persécuter si elles venaient à découvrir la nature tenue pour répugnante de son vice caché. Dans son ouvrage Hiding from Humanity: Disgust, Shame and the Law, Martha C. Nussbaum argumente que le dégoût est un refus d'ingérer et que, par conséquent, il suggère aussi un refus d'être contaminé12. Avec la maturité et le recul que lui accordent les soixante années qui se sont écoulées depuis cette période de jeunesse hantée par la peur, Léo se rappelle à quel point il a eu du mal à concilier son orientation sexuelle avec la loi morale de la Heimat, confusion de ses sentiments qui le laisse déchiré entre le désir et la répulsion.

Ce déchirement entre désir et refoulement devient surtout palpable dans la description que Léo fait d'un lieu qu'il a fréquenté à maintes reprises dans sa ville natale avant de se voir déporté dans le camp de

11. - « Mir war bereits etwas zugestoßen. Etwas Verbotenes. Es war absonderlich, dreckig, schamlos und schön. » (AS, 8).

12. - «Dans toutes les sociétés, cependant, le dégoût exprime un refus d'ingérer et donc d'être contaminé par ce qui évoque l'idée de sa propre mortalité et de sa propre décadence en tant qu'espèce animale. » «In all societies, however, disgust expresses a refusal to ingest and thus be contaminated by a potent reminder of one's own mortality and decay-prone animality. » Martha C. Nussbaum, Hiding from Humanity: Disgust, Shame and the Law, Princeton, Princeton UP, 2004, p. 97. 
travaux forcés. Ce lieu de leurre est un pavillon dans le parc municipal, un endroit où se sont déroulés plusieurs de ses rendez-vous avec d'autres hommes. Sa description du pavillon semble refléter les sentiments de confusion qu'il éprouvait par rapport à son homosexualité, de même que la répulsion anticipée de son entourage :

En rentrant chez moi, j'étais allé au milieu du parc, sous le kiosque où des orchestres jouaient les jours fériés. Je m’y étais assis un moment. La lumière perçait à travers le bois ajouré. Je voyais l'anxiété des cercles, des carrés et des losanges évidés, reliés par des plantes grimpantes et leurs vrilles blanches. Leur motif était celui de mon égarement, et de l'épouvante sur le visage de ma mère ${ }^{13}$. (BS, 10)

Dans le contexte de la Heimat, ces sentiments de confusion et de répulsion que le jeune homme associe à la perception que sa famille, sa communauté et lui-même se font de l'homosexualité correspondent donc aussi au refus idéologique de se voir contaminé moralement. De telles contaminations morales seraient perçues comme abjectes et pourraient s'avérer une menace pour la pureté et l'innocence de la Heimat. L'homosexualité, dans ce contexte, devient alors une condition d'anormalité et de disgrâce, une orientation sexuelle qui va à l'encontre du naturel et donc de la nature, si chère et précieuse aux yeux de celles et ceux qui adhèrent au concept identitaire ancré dans l'espace et les valeurs traditionnelles. Conscient de son manque de vertu morale, Léo perçoit donc son exploration sexuelle, c'est-à-dire la période où " [il] était [le gibier] qu'on se repassait » (BS, 11) et qu'il définit de «Wildwechsel », d'échange bestial (AS, 8), comme une sorte de promiscuité à la fois clandestine et sauvage. Son impression de se livrer à des actes qu'il associe à la déviance morale est ce qui le pousse à percevoir son homosexualité comme étant un travers, une tare qu'il doit cacher à tout prix et garder sous silence. Mais justement, ce secret, qu'il apprend à soigner et à protéger dans ses efforts de clandestinité, pourrait justifier la raison pour laquelle, parmi l'énumération d'adjectifs qu'il associe à la nature immorale et taboue de ses désirs, tels que «bizarre, sale, impudique »14 (BS, 10), on retrouve également l'adjectif «beau » pour décrire le plaisir que lui procure le désir de l'interdit.

En se remémorant les rencontres secrètes qui l'ont laissé confus, Léo se souvient d'avoir participé à ce qu'il appelle des « rendez-vous », ce

13. - « Auf dem Heimweg bin ich in die Parkmitte, in den runden Pavillon gegangen, wo an Feiertagen die Orchester spielten. Ich blieb eine Weile darin sitzen. Das Licht stach durchs feingeschnitzte Holz. Ich sah die Angst der leeren Kreise, Quadrate und Trapeze, verbunden durch weiße Ranken mit Krallen. Es war das Muster meiner Verirrung und das Muster des Entsetzens im Gesicht meiner Mutter» (AS, 8).

14. - «absonderlich, dreckig, schamlos » (ibid.). 
terme utilisé pour faire référence aux rencontres anonymes auxquelles il s'est adonné avec des hommes de sa Heimat avant sa déportation, malgré le risque qui le guettait déjà à l'époque de ses 17 ans. Or, le sentiment de se livrer à des jeux interdits semble avoir ajouté de l'engouement à sa dépendance homo-érotique, si l'on se réfère à ses propres mots : « À force de m'en empêcher, je me suis hâté d'y retourner [...] Au rendezvous, comme on disait au parc $»^{15}(\mathrm{BS}, 10)$. Dans la version originale, ce terme français utilisé en contexte germanophone ajoute une dimension secrète aux rencontres sexuelles furtives et clandestines auxquelles Léo se livre et qu'il décrit dans le passage suivant :

J'ai eu un second rendez-vous avec le premier homme, le même. Il s'appelait l'HIRONDELLE. Le deuxième était nouveau, LE SAPIN. Le troisième s'appelait L'OREILLE. Ensuite, il y a eu LE FIL, puis LE LORIOT, LE BONNET. Plus tard, LE LIÈVRE, LE CHAT, LA MOUETTE. Et LA PERLE. Nous étions les seuls à savoir qui ces noms désignaient ${ }^{16}$. (BS, 10-11)

Bien que les pseudonymes semblent aléatoires, tous ont une seule et même fonction : conserver l'anonymat et camoufler une action, une identité interdite. Rongé par un sentiment de culpabilité, Léo - et par extension ses partenaires sexuels - est conscient du risque qui le guette sous le « generalized ocular regime »17 de la Heimat, ce « dé à coudre [,] où toutes les pierres avaient des yeux » (BS, 7). Obsédé et menacé par la surveillance de la collectivité, son désir de fuir la Heimat ne cesse de croître.

\section{Les frontières de l'inclusion et de l'exclusion de la Heimat}

Se sachant la proie des mécanismes de surveillance de la Heimat, Léo a conscience d'être menacé et troublé par les frontières à la fois physiques et idéologiques qui l'entourent, que celles-ci soient d'ordre local, régional ou national. Au-delà de sa petite ville natale, c'est tout un pays qui rejette et criminalise l'homosexualité. Celle-ci est d'ailleurs interdite par la loi en Roumanie de 1864 à 2001. Dans le contexte germano-roumain de la Transylvanie, région natale du protagoniste, les lois anti-homosexuelles du Troisième Reich, connues sous les noms de Paragraph 175 et Paragraph 175a et appliquées à travers le terri-

15. - « Je mehr ich mich davon abhielt, desto schneller ging ich wieder hin - nach zwei Tagen. Zum Rendezvous, so hieß das im Park. » (ibid.).

16. - « Ich ging zum zweiten Rendezvous mit demselben ersten Mann. Er hieß DIE SCHWALBE. Der zweite war ein neuer, er hieß DIE TANNE. [...] Danach kam DER FADEN. [...] Dann DIE PERLE. Nur wir wussten, welcher Name zu wem gehört. » (ibid.).

17. - G. Shapiro, Archeologies of Vision, op. cit., p. 8. 
toire occupé, ont également infiltré le discours moral et normatif des communautés germanophones de Roumanie, dont plusieurs de ses membres se sont ralliés aux idéologies et aux régiments nazis pendant la guerre. Qui plus est, l'entourage du jeune homme semble avoir admiré et soutenu le régime de Hitler, tel que suggéré dans le passage suivant : « Et des voisins, des parents et des professeurs partaient à la guerre rejoindre les fascistes roumains ou Hitler $»^{18}$ (BS, 65). La probabilité que les lois anti-homosexuelles aient pu jouer un rôle d'importance dans le discours moral et normatif de son entourage est donc d'autant plus vraisemblable puisque, à en croire ses propos, ses propres parents adhéraient fièrement et ouvertement aux idéologies nazies, notamment à celles ayant trait aux discours sur la supériorité de la race aryenne. C'est dans ce même contexte qu'il se remémore la manière dont ses parents ont interféré dans son développement physique, afin de mieux respecter les standards de la Heimat en matière d'idéal masculin : «Et une fois par semaine, comme j'étais grassouillet et qu'il fallait avoir une allure martiale, ils me faisaient suivre un cours privé de gymnastique, le cours des nabots »19 (BS, 62). Tel qu'exposé dans ce passage, les leçons particulières de sport, qu'il appelle «Krüppelturnen » (AS, 54), le cours des nabots, sont organisées pour cultiver et masculiniser les jeunes qui sont perçus comme « invalides », ne serait-ce que de par leur manque de tonus musculaire, ou encore en raison de leur excès de poids. Léo est victime de regards désapprobateurs de sa famille et de ses proches en raison de sa silhouette non conforme et de son manque d'athlétisme, ce qui ne fait qu'amplifier ce sentiment d'être surveillé et jugé par ceux qui l'entourent. Déjà conscient d'enfreindre les normes physiologiques prônées par son entourage, il voit son secret peser encore plus lourdement contre lui.

À défaut de pouvoir honorer les standards hétéro-normatifs imposés par le discours local, le narrateur se voit obsédé par l'opinion de son environnement social. Le regard désapprobateur qu'il pose sur luimême le pousse à imaginer le sort qui pourrait lui être réservé s'il devait effectivement se trouver «PRIS EN FLAGRANT DÉLIT $»^{20}$ (BS, 12), tel qu'il le décrit dans le passage suivant :

Comme tous les Allemands de notre petite ville, ma mère et surtout mon père croyaient à la beauté des nattes blondes et des chaussettes blanches. Au rectangle noir de la moustache d'Hitler, et à nous, les

18. - « Und es gingen Nachbarn und Verwandte und Lehrer in den Krieg zu den rumänischen Faschisten oder zum Hitler. » (AS, 56).

19. - « Und mich schickten die Eltern, weil ich zu pummelig war und soldatischer werden sollte, einmal pro Woche zum privaten Turnunterricht, dem Krüppelturnen » (AS, 54).

20. - «AUF FRISCHER TAT ERTAPPT » (AS, 9). 
Saxons de Transylvanie, de race aryenne. Sur le plan strictement physique, mon secret était une énorme turpitude. Si c'était avec un Roumain, l'infamie raciale venait s'y ajouter ${ }^{21}$. (BS, 13)

En se fiant à cette observation, avoir une relation intime avec un Roumain serait, à son avis, perçu comme un vice d'ordre ethnique par rapport aux normes raciales imposées par sa communauté germanophone. Ayant déjà rompu avec les lois de la Heimat en ce qui a trait aux idéologies physiques et raciales, Léo perçoit son homosexualité non pas simplement comme une entrave aux normes de la Heimat, mais déjà comme étant la source de toute son angoisse, cette peur constante et envahissante qui le tourmente et l'étouffe. Cette angoisse - qui le désemparait déjà à l'époque de ses 17 ans - continue de le hanter soixante ans plus tard : " Je porte des bagages qui ne font pas de bruit. Depuis bien longtemps, mon bagage de silence est si profond que je ne pourrai jamais tout déballer. Quand je parle, je ne fais que m'emballer dans un autre bagage de silence ${ }^{22}$ (BS, 12). Que ce soit chez lui dans la Heimat ou lors de sa déportation dans le Lager, le silence devient ce refuge invoqué pour éviter la honte et assurer ses chances de survie.

\section{Ce qui brille voit, ou la hantise du Lager et de la Heimat}

L'optimisme de Léo, ou plutôt la naïveté de ce dernier au moment d'être déporté au Lager, en janvier 1945, lui a donné l'espoir illusoire qu'il pourrait, une fois déporté, se libérer du panoptique de sa ville. Et pourtant, le risque d'être pris en flagrant délit n'aura fait qu'empirer durant ses années d'incarcération au camp de travaux forcés : « À l'époque du camp, si je m'étais fait pincer sur place, on m'aurait tué »23 (BS, 12). Après avoir raconté au lecteur les horreurs, mais aussi les moments de solidarité entre incarcérés qu'il a connus durant ces cinq années passées au camp de travaux forcés, le narrateur fait part de l'expérience elle aussi traumatisante de revenir à la réalité dans sa ville natale, et ce après avoir survécu à l'horreur de la famine et de la brutalité carcérale. Bien que libérée physiquement du camp, son âme, elle, demeure hantée par le traumatisme laissé par le Lager, ce lieu d'anéantissement de la condi-

21. - « Meine Mutter und besonders mein Vater glaubten, wie alle Deutschen in der Kleinstadt, an die Schönheit blonder Zöpfe, weißer Kniestrümpfe. An das schwarze Viereck von Hitlers Schnurrbart und an uns Siebenbürger Sachsen als arische Rasse. Mein Geheimnis war, rein körperlich betrachtet, schon höchste Abscheulichkeit. Mit einem Rumänen kam noch Rassenschande dazu » (AS, 10-11).

22. - « Ich trage stilles Gepäck. Ich habe mich so tief und so lang ins Schweigen gepackt, ich kann mich in Worten nie auspacken. Ich packe mich nur anders ein, wenn ich rede. » (AS, 9).

23. - « Und in der Lagerzeit - im Lager erwischt, wär ich tot gewesen. » (Ibid.). 
tion humaine où l'existence même de l'homme bascule entre le permis et l'interdit.

Au bout de ces cinq années dans le camp, et donc de retour dans son pays natal, Léo se sent à nouveau menacé et surveillé, et par conséquent déconnecté et isolé de son environnement social, cette Heimat qui continue de l'exclure et de le terroriser : « [...] je rôdais jour après jour dans le tumulte des rues en répétant dans ma tête les phrases les meilleures en cas d'arrestation. PRIS EN FLAGRANT DÉLIT : contre cette sentence de culpabilité, je me forgeais des centaines de prétextes et d'alibis $»^{24}$ (BS, 12). Contrairement au confinement physique que représentait le Lager, il se voit étourdi et apeuré par la liberté soudaine qu'il a retrouvée. Ce retour à la normalité le rend nostalgique de l'identité qu'il s'était forgée durant ces cinq années de confinement dans le Lager : " J'étais enfermé en moi et j'en étais expulsé, je ne leur appartenais pas et je me manquais à moi-même $»^{25}(\mathrm{BS}, 321)$. Car cette identité du « Nichtrührer ${ }^{26}$ - pseudonyme qui met en valeur le stoïcisme infaillible dont il s'était muni pour éviter la mort certaine qui le guettait dans le Lager - lui avait somme toute permis de vivre des moments de complicité et de solidarité malgré toute l'horreur endurée. De retour dans la Heimat, lieu qu'il perçoit comme étant à la fois étrange et familier, il est de nouveau hanté par les démons du passé, ces vieilles habitudes compulsives auxquelles il fut en mesure de mettre fin temporairement durant la période au camp. Que ce soit avant, durant ou après la déportation, le poids de son secret continue de peser sur lui :

Avant, pendant et après le camp, pendant vingt-cinq ans, j'ai vécu dans la peur de l'État et de ma famille. Je redoutais une double déchéance : d'être criminel incarcéré par l'État, et d'être la honte de la famille, qui m'exclurait ${ }^{27}$. (BS, 12)

Sa peur d'être mis à nu, cette angoisse d'être doublement déchu - par l'État d'une part, puis par sa famille de l'autre - devient insoutenable lorsqu'il se voit refranchir les portes du Neptunbad; ces bains publics

24. - « Ich streifte nach den fünf Lagerjahren Tag für Tag durch den Tumult der Straßen und übte im Kopf die besten Sätze für den Fall meiner Verhaftung: AUF FRISCHER TAT ERTAPPT » (Ibid.).

25. - « Ich war eingesperrt in mich und aus mir herausgeworfen, ich gehörte nicht ihnen und fehlte mir » (AS, 272).

26. - Rendu par « l'avachissement » dans la traduction française. Cette traduction ne transmet pas cette idée de distance, voire de secret que Müller associe au terme Nichtrührer dans le texte original (BS, 311).

27. - « Vor, während und nach meiner Lagerzeit, fünfundzwanzig Jahre lang habe ich in Furcht gelebt, vor dem Staat und vor der Familie. Vor dem doppelten Absturz, dass der Staat mich als Verbrecher einsperrt und die Familie mich als Schande ausschließt. » (AS, 10). 
qui, tout comme le pavillon du parc, servaient d'endroit clandestin pour les rencontres intimes entre hommes. Leurré par ces bains publics, il se rappelle y avoir remis les pieds instinctivement et sans aucune hésitation : «Sans réfléchir, je poussai la porte et entrai dans le hall. Le dallage sombre miroitait comme une eau calme. Sous moi, je vis mon manteau clair flotter vers la caisse. Je demandai un billet d'entrée ${ }^{2} 8$ (BS, 338). Incapable de résister à la tentation, il se sentit tel un automate, guidé par l'impulsion, conscient d'être potentiellement tombé dans le piège d'être pris sur le fait :

La caissière demanda : un ou deux.

J'espérai que sa bouche exprimait une illusion d'optique, et non des soupçons. J'espérai qu'elle ne voyait pas mon double manteau, ni que j'étais en train de revenir à mon ancienne vie ${ }^{29}$. (BS, 338)

Pensant avoir été découvert par la caissière dont les mots semblent suspects et truffés de sous-entendus, Léo croit pour un instant s'être fait prendre par les mécanismes de surveillance de ce lieu clandestin, lorsqu'il observe : « La caissière était nouvelle, mais le hall, le sol brillant, la colonne centrale, la vitre plombée de la caisse et le carrelage aux nénufars me reconnaissaient $»^{30}$. Ainsi, le doute qui s'est installé chez lui suite à son contact avec la caissière ne fut qu'accru par le caractère panoptique qu'il associe à ce lieu hanté par l'interdit qu'était le Neptunbad : « [ce] décor froid [qui] avait sa propre mémoire [et dont] les ornements [luisants] n'avaient pas oublié qui [il] étai[t] ${ }^{31}$.

Les éléments luisants dont se souvient le narrateur indiquent une certaine intertextualité avec d'autres éléments brillants que l'on peut associer à la surveillance dans les récits de Müller, notamment dans son roman Le renard était déjà le chasseur (1992). Tout comme la protagoniste Adina, dont les convictions politiques font d'elle la cible des regards désapprobateurs d'une dictature roumaine qu'elle repère sous la devise métonymique « was glänzt, sieht » (ce qui brille voit) ${ }^{32}$, Léo

28. - « Ohne zu überlegen, ging ich durch die Tür in die Halle. Der dunkle Steinboden spiegelte wie ruhiges Wasser. Ich sah meinen hellen Mantel unter mir zur Kassenloge schwimmen. Ich verlangte eine Karte » (AS, 286).

29. - « Die Kassenfrau fragte: Eine oder zwei.

Hoffentlich sprach aus ihrem Mund nur die optische Täuschung, nicht ein Verdacht. Hoffentlich sah sie nur den doppelten Mantel und nicht, dass ich unterwegs war in mein altes Leben » (ibid.).

30. - «Die Kassenfrau war neu. Aber die Halle erkannte mich, der blanke Boden, die Mittelsäule, die Bleiverglasung am Schalter, die Kachelwände mit dem Seerosenmuster » (ibid.).

31. - « Der kalte Schmuck hatte sein eigenes Gedächtnis, die Ornamente hatten nicht vergessen, wer ich bin. » (AS, 286-287).

32. - Herta Müller, Le renard était déjà le chasseur, roman traduit de l'allemand par Claire de Oliveira, Paris, Seuil, 1997, p. 28. 
associe lui aussi les éléments luisants du Neptunbad à des mécanismes de surveillance. Par conséquent, il perçoit ces bains publics comme étant un lieu converti en guet-apens, dont la communauté se sert pour exposer des hommes qui, comme lui, vivent dans l'interdit et la clandestinité. Suite à cet épisode frôlant la paranoïa, le jeune homme prend le contrôle de ses désirs et de ses pulsions pour éviter d'être «PRIS EN FLAGRANT DÉLIT » (BS, 10).

Voulant se conformer aux règles de la Heimat, il se plie à la normativité et finit par épouser Emma, une fille qu'il rencontre peu de temps après sa mésaventure dans le hall d'entrée des bains publics. En épousant celle-ci, il se voit adhérer à l'institution hétéro-normative du mariage, ce qui, à son avis, peut l'aider dans ses démarches, voulant conserver une façade de normalité aux yeux des membres de sa communauté. Et pourtant, malgré l'amitié qui l'unit à Emma, le mariage n'aura été justement qu'une simple façade. Devant camoufler sa véritable identité à son épouse, le narrateur se rappelle avoir été accablé plus que jamais par le poids de son secret et de sa honte. Cela deviendra encore plus palpable dans le chapitre $"$ Je suis toujours le piano ${ }^{33}$ (BS, 335), dans lequel il se remémore cette sortie au restaurant où il fut soudainement pris de panique lorsque le serveur, prenant leur commande, fit un commentaire observateur par rapport au pianiste du restaurant qui jouait sur un instrument désaccordé : « vous entendez, le piano joue faux, je l'ai pourtant bien dit au patron. Et il a trouvé le moyen de virer le pianiste ${ }^{34}$ (BS, 340). Pour Léo, les mots « le piano joue faux » ne sont pas tant un reproche vis-à-vis des fausses notes du piano qu'une critique envers son propre jeu de cache-cache lié à sa double identité. Sa crainte d'être pris en flagrant délit semble se confirmer lorsque son épouse Emma ajoute ses propres propos insinuants à ceux du serveur : "Ben tu vois, c'est toujours sur le musicien que ça tombe, jamais sur le piano »35 (BS, 341). Tout comme la situation vécue avec la caissière au Neptunbad, il se sent exposé par sa conjointe Emma, dont les mots semblent truffés de sousentendus voulant dévoiler son identité cachée et refoulée. Cette peur d'avoir été pincé plonge Léo dans la consternation et la paranoïa:

Pourquoi avait-elle attendu le départ du serveur pour lancer cette phrase. J'espérais qu'elle ne savait pas ce qu'elle disait. Au parc, mon nom de code était LE MUSICIEN, à l'époque. La peur est implacable.

33. - « Ich bin noch immer das Klavier» (AS, 284).

34. - «Da hören Sie es, das habe ich doch dem Chef die ganze Zeit versichert, das Klavier spielt falsch. Und was hat er gemacht, er hat den Pianisten rausgeschmissen. » (AS, 288).

35. - « Na siehst du, immer erwischt es den Spieler, nie das Klavier. » (AS, 289). 
Je changeai de parc et de nom d'emprunt. Au nouveau parc, loin de chez moi et proche de la gare, je choisis de m'appeler LE PIANO ${ }^{36}$ (BS, 341).

Ses doutes se renforcent lorsqu'il se sent soudainement scruté et jugé par sa conjointe. Décrivant son regard à la fois perçant et provocateur, Léo demeure hanté par la scène suivante : « Dans ses yeux tournaient des roues dentées de couleur jaune. Elles étaient rouillées, et les paupières y restaient accrochées à chaque battement ${ }^{37}$ (BS, 341). Près de soixante années plus tard, n'ayant toujours pas oublié le regard jaune et rouillé de son épouse, il reste d'avis que les yeux de celle-ci étaient, à son plus grand effroi, révélateurs des mécanismes de sa pensée. Pour lui, le regard inquisiteur d'Emma fut le signe évoquant le fait que, désormais, elle était au courant de son secret et que, par conséquent, elle refusait de demeurer aveugle devant la vérité, c'est-à-dire devant l'identité homosexuelle refoulée de son époux. Dès lors, il réalise qu'il ne peut plus vivre avec ce mensonge envers Emma et envers lui-même, ce faux mariage qu'il aura simulé pendant près de onze années de vie conjugale.

Voulant mettre fin au cercle vicieux des mensonges - qui, jusque-là, lui avaient permis de cacher sa véritable identité -, le narrateur choisit pourtant de mentir une dernière fois à Emma en l'informant de son désir de rendre visite à sa tante à Vienne, alors même qu'il sait qu'il ne reviendra plus. Sa décision finale de tout quitter sera précipitée par le danger imminent de dénonciation qui le guettait et qui se produisait dans son entourage :

À cette époque-là, au parc, LE COUCOU et LA TABLE DE NUIT s'étaient fait arrêter. Je savais qu'une fois au commissariat, presque tout le monde dénonçait les autres, et que je n'aurais aucun moyen de m'en tirer si on mentionnait LE PIANO. Je demandai une autorisation de visite en Autriche ${ }^{38}$ (BS, 342).

Pour Léo, qui avait déjà connu les souffrances de la déportation, l'exil devient la seule issue de secours. Son Besuchsantrag, sa demande

36. - « Wieso hatte sie mit diesem Satz gewartet, bis der Kellner weg war. Ich hoffte, sie weiß nicht, was sie sagt. Im Park habe ich damals den Decknamen DER SPIELER gehabt. Angst kennt kein Pardon. Ich habe den nahen Park gewechselt. Und meinen Decknamen. Für den neuen Park weit von der Wohnung und nahe am Bahnhof habe ich mir den Namen DAS KLAVIER genommen. » (ibid.).

37. - « In ihren Augen drehten sich gelbe Zahnrädchen. Sie waren angerostet, ihre Lider blieben beim Blinzeln daran hängen. » (ibid.).

38. - «Zu derZeit waren im Park DER KUCKUCK und DAS NACHTKÄSTCHEN verhaftet worden. Ich wusste, dass bei der Polizei fast alle reden und mir keine Ausrede etwas nützt, wenn die zwei DAS KLAVIER erwähnen. Ich stellte einen Besuchsantrag für Österreich. » (AS, 290). 
de visite pour l'Autriche, lui permet enfin de fuir le panoptique de sa Heimat.

\section{Conclusion}

Après des années à passer son identité sous silence, à craindre la menace, la punition et l'exclusion, Léo choisit de quitter la peur et la hantise de cette Heimat qu'il perçoit comme étant un lieu « où toutes les pierres avaient des yeux » (BS, 10). Ce sentiment de confinement claustrophobique ressenti par Léo dans sa famille et dans sa ville natale, et qui découle de la peur d'être scruté, exposé et condamné pour son homosexualité secrète, explique en partie pourquoi ceux qui, comme Léo, échappent aux menaces de la surveillance choisissent de rompre tous les liens affectifs avec leur Heimat. Mais la hantise, elle, demeure. Bien qu'il soit en mesure d'éviter d'être pris au piège, humilié et sanctionné pour sa véritable identité, Léo le narrateur confie, soixante années plus tard, qu'il demeure hanté par le sentiment de culpabilité d'avoir lâchement trahi son épouse Emma :

C'est ma plus grande faute, à ce jour. Je me suis travesti pour un voyage censé être bref et, avec une valise légère, je suis allé en train à Graz, d'où j'ai écrit une carte qui tenait dans la main. Chère Emma,

La peur est implacable.

Je ne reviendrai pas $^{39}$ (BS, 343).

Pour Léo, «Je ne reviendrai pas » (BS, 343) est la phrase qui met fin à l'hypocrisie de son mariage, ce semblant de normalité dont le but fut de servir l'intérêt de la normativité. Et pourtant, ces derniers mots adressés à sa femme lui permettent de se libérer du poids étouffant d'une fausse identité, mais aussi du panoptique de la Heimat, ce lieu que Müller dépeint encore une fois comme étant hanté par l’interdit.

39. - «Es ist bis heute meine schwerste Schuld, ich habe mich für eine kurze Reise kostümiert, bin mit einem leichten Koffer in den Zug gestiegen und nach Graz gefahren. Von dort habe ich eine handgroße Karte geschrieben:

Liebe Emma,

Angst kennt kein Pardon.

Ich komme nicht wieder. » (AS, 291). 